\title{
EP-112
}

\section{Volvulus of the gallbladder: Beware of aberrant biliary anatomy}

\author{
Dhanushke FERNANDO*, Suresh NAVADGI, Ruwan WIJESURIYA
}

Department of General Surgery, St. John of God Midland Public \& Private Hospitals, Australia

Introduction: Gallbladder volvulus (GBV) is an uncommon differential in the acute surgical abdomen, which may incur significant morbidity if missed. Less than 500 cases have been reported in the literature, with few diagnosed pre-operatively. It is defined as the rotation of the gallbladder on its mesentery along the axis of the cystic pedicle, although cases of torsion of the gallbladder fundus itself have been reported.

Methods: An 82-year-old lady presented with a worsening 5 day history of right upper quadrant pain with associated nausea, anorexia. She had worsening pain and noticed a 'lump' in her abdomen, along with subjective fevers. On examination, her abdomen was tender, with a non-pulsatile mass in the RUQ with voluntary guarding. The computed tomography Abdo-Pelvis revealed a distended gallbladder extended up to the pelvis with multiple gallstones. Stranding of fat around the gallbladder was noted, with wall thickening up to $7 \mathrm{~mm}$ at the fundus - with overall appearances consistent with acute cholecystitis.

Results: An open cholecystectomy was performed, with the intraoperative finding of GBV. There were multiple small arteries supplying the gallbladder, with no definitive cystic artery. The extra-hepatic biliary anatomy was aberrant with a long-cystic duct exiting through the cystic plate. Post-operatively recovery was uncomplicated.

Conclusions: GBV is an important differential of RUQ pain, requiring immediate intervention. It is often misdiagnosed as cholecystitis. Imaging is variable in detecting GBV, and early surgical intervention remains the mainstay of both diagnosis and treatment. However, surgeons should beware that unrecognized aberrant biliary anatomy can result in higher morbidity. 